\title{
Minimally Invasive Surgery in Treatment of Traumatic Thoracolumbar Spinal Injuries: A 2-Year Experience
}

Kow Ren $\mathrm{Yi}^{1}$, Ed Simor Khan Mor Japar Khan ${ }^{1}$, Fauziana Abd Jabar ${ }^{2}$, Su Yuen², Low Chooi Leng ${ }^{3}$

${ }^{1}$ Department of Orthopaedic Surgery, International Islamic University Malaysia

2Department of Orthopaedic Surgery, Hospital Tengku Ampuan Afzan, Kuantan

Pahang

32Department of Radiology, Hospital Tengku Ampuan Afzan, Kuantan Pahang.

Introduction: Majority of the traumatic spine injuries are located at the thoracolumbar region. They can be compression fractures, burst fractures, flexionextension injuries (Chance fractures), dislocations, or any of the combination. Surgery is indicated for patients with thoracolumbar injury classification scoring (TLICS) of 4 or more. Traditionally, surgical approaches for thoracolumbar spinal injuries involve open surgery, instrumentation with/without decompression. In our previous study, open surgery for traumatic thoracolumbar injuries and history of blood transfusion have been found to be associated with higher risk of deep surgical site infection requiring surgical debridement. With the advent of modern implants and equipments, minimally invasive surgery (MIS) has been made possible for spine surgeries. We report our two-year experience in utilizing MIS to treat traumatic thoracolumbar spinal injuries. Materials and method: Patients who underwent spinal surgeries at Hospital Tengku Ampuan Afzan, Kuantan from July 2017 to July 2019 were screened for suitability to be included in this study. Only patients who underwent spinal minimally invasive surgeries have been included in this study. Patients who underwent open spinal surgeries were excluded. Results: A total of 8 patients were included in this study. There were 3 burst fractures and 5 chance fractures. All patients underwent a minimum of 4-level posterior spinal instrumented fusion with MIS techniques and 2 patients had laminectomy at the injured level for decompression. All but one patient did not require blood transfusion and there was no incidence of surgical site infection among these patients. Conclusion: Minimally invasive surgery (MIS) is a better option compared to open surgery in treating traumatic thoracolumbar spinal injuries. By minimizing the surgical incision, we are able to reduce blood loss and avoid deep surgical site infection. 